\title{
Anos potenciais de vida perdidos por intoxicação exógena no Brasil no período de 2007 a 2017
}

\author{
Potential years of lives lost from exogenous intoxication \\ in Brazil from 2007 to 2017
}

\section{Sheila Silva Maia', Verine Silva de Souza², Emanuela Dias Souza³, Tássia Nery Faustino ${ }^{4}$}

1Autora para correspondência. Fundação de Hematologia e Hemoterapia da Bahia. Salvador, Bahia, Brasil. ORCID: 0000-0003-1029-5554. sheu-maia@hotmail.com 2UPA 24 h Lucas Evangelista. Salvador, Bahia, Brasil. ORCID: 0000-0002-2375-4126. verine.enf@gmail.com 3HAPVIDA. Salvador, Bahia, Brasil. ORCID: 0000-0002-9397-8651. dias.emanuela@hotmail.com ${ }^{4}$ Universidade do Estado da Bahia. Salvador, Bahia, Brasil. ORCID: 0000-0001-7854-4540. tassiafaustino@yahoo.com.br

RESUMO | OBJETIVO: Estimar os Anos Potenciais de Vida Perdidos (APVP) por intoxicação exógena no Brasil entre os anos de 2007 a 2017. MÉTODO: Estudo descritivo e exploratório, com dados secundários obtidos através do Sistema Nacional de Agravos de Notificação (SINAN) e do Sistema de Informação sobre Mortalidade (SIM), de 2007 a 2017, no Brasil. As variáveis investigadas foram: óbitos por intoxicação exógena, faixa etária (15-19 anos, 20-39 anos, 4059 anos, 60-64 anos e 65-69 anos), agente tóxico, ano, sexo e região do país. RESULTADOS: Verificou- se um total de 317.687 APVP, onde a faixa etária entre 20 a 39 anos representa $56 \%$ dos óbitos, com predomínio no sexo masculino, correspondendo a $60 \%$ dos casos de morte prematura. 0 uso indevido de medicamentos foi responsável pelo maior número de APVP (74.131). A região sudeste apresentou maior valor de APVP que outras regiões do país. CONCLUSÃO: As intoxicações exógenas foram responsáveis por importante parcela de mortes prematuras e de APVP segundo a análise da mortalidade proporcional, sendo maior em indivíduos que fazem uso indevido de medicamentos, do sexo masculino, entre 20 e 59 anos e moradores do sudeste brasileiro.

PALAVRAS-CHAVE: Intoxicação. Óbito. Registros de mortalidade. Anos Potenciais de Vida Perdidos. Sistema de Informação em Saúde.

\begin{abstract}
OBJECTIVES: This work aims to assess the Potential Years of Life Lost (PYLL) according to causes of exogenous poisoning in Brazil (2007-2017). METHOD: Descriptive and exploratory study, with secondary data obtained from the relevant national institutions, the Sistema Nacional de Agravos de Notificação (SINAN) and the Mortality Information System (SIM), from 2007 to 2017, in Brazil. The variables investigated were: deaths by intoxication; groups aged 15 to 19 years old, 20 to 39, 40 to 59,60 to 64 and 65 to 69; toxic agent; year; gender and each region of the country. RESULTS: There were a total of 317,687 PYLL, in which the age group aged 20 to 39 years old represented $56 \%$ of the deaths, with male prevalence corresponding to $60 \%$ of cases of premature death. Undue use of medicines accounted for the highest number of PYLL $(74,131)$. The Southeastern Region presented a higher PYLL value than other regions of the country. CONCLUSION: Exogenous intoxications were responsible for a significant portion of premature deaths and PYLL according to proportional mortality analysis. Most of the deaths, according to groups of causes analyzed on this work, were male deaths aged 20 to 59 years old, who were users of undue medicines, Southeastern Brazil' local residents.
\end{abstract}

KEYWORDS: Poisoning. Death. Mortality records. Potential Years of Life Lost. Health Information System. 


\section{Introdução}

Intoxicação exógena é o conjunto de efeitos nocivos caracterizados por manifestações clínicas ou laboratoriais que revelam o desequilíbrio orgânico produzido pela interação de um ou mais agentes tóxicos com o sistema biológico ${ }^{1}$. Esse evento é considerado um problema significativo de saúde pública global. Conforme dados da Organização Mundial de Saúde (OMS), em 2012, estima-se que 193.460 pessoas morreram em todo o mundo devido a envenenamento não intencional. Destas mortes, $84 \%$ ocorreram em países de baixa e média renda. No mesmo ano, o envenenamento não intencional causou a perda de mais de 10,7 milhões de anos de vida saudável ${ }^{2}$.

A intensidade da ação do agente tóxico é proporcional à concentração e ao tempo de exposição. As intoxicações por substâncias químicas podem ser agudas ou crônicas e poderão se manifestar de forma leve, moderada ou grave, a depender da quantidade da substância química absorvida, do tempo de absorção, da toxicidade do produto, da suscetibilidade do organismo e do tempo decorrido entre a exposição e o atendimento médico ${ }^{3}$.

De acordo com o DATASUS foram registrados 605.353 óbitos por intoxicação exógena no Brasil em 2017, e destes, $64 \%$ ocorreram na região sudeste. São Paulo é destaque com $32 \%$ dos registros de óbitos por intoxicação. No país, os casos de intoxicação acometem mais frequentemente as faixas etárias entre 20 a 39 anos, e com predominância do sexo masculino, que representa aproximadamente $60 \%$ dos $\operatorname{casos}^{4}$.

Diante disso, é imprescindível avaliar a condição de saúde de uma população, e esta, perpassa pela determinação das doenças e agravos que mais acarretam mortalidade. Por conseguinte, não somente a taxa de mortalidade revela tal conjuntura, sendo necessário avaliar outros parâmetros como magnitude, vulnerabilidade e transcendência. A quantidade de óbitos relacionada à determinada causa demonstra sua magnitude. Já a vulnerabilidade do dano é a capacidade operacional de reduzir o dano, enquanto que a transcendência é o valor social dado ao problema 5 .

Os Anos Potenciais de Vida Perdidos (APVP) é o indicador que viabiliza informações tanto da extensão da mortalidade na população, descrita por meio das taxas de mortalidade e da mortalidade proporcional, quanto quantifica o impacto resultante desta perda para a sociedade, qualificando estas mortes ${ }^{6}$.

Por conseguinte, a mortalidade prematura se destaca não só pelo impacto relacionado ao valor social do óbito, mas também pelo potencial dos anos de vida produtivos que são perdidos. Tal situação não afeta somente o indivíduo, mas também a coletividade que é privada do seu potencial intelectual e econômico ${ }^{5}$.

Sabe-se que a análise dos dados de intoxicação é indispensável para a tomada de decisões em favor da melhoria da condição de saúde da população. Mesmo assim, a subnotificação é um acontecimento frequente nos bancos de dados e é visível nos gráficos que retratam a intoxicação exógena no Brasil, pela ausência das informações no Sistema Nacional de Informações Tóxico-Farmacológicas (SINITOX), tanto em regiões do país como o sul e o norte, como em estados de regiões do nordeste, sudeste e centro-oeste. É desconhecido em 2016, por exemplo, os dados referentes ao desequilíbrio orgânico causado por agentes tóxicos na Bahia.

Contudo, apesar do expressivo número de óbitos por intoxicação exógena no Brasil e do impacto familiar, social, econômico e cultural gerado pela perda humana prematura, verifica-se, através de pesquisa eletrônica realizada na base de dados da Biblioteca Virtual em Saúde, a ausência de estudos voltados para a análise dos APVP por intoxicação no país. Face às considerações realizadas, esse estudo apresenta como objetivo geral estimar os anos potenciais de vida perdidos por intoxicação exógena no Brasil entre os anos de 2007 a 2017 e como objetivos específicos: determinar as taxas de mortalidade proporcional por ano e identificar os anos potenciais de vida perdidos por faixa etária, agente causal, sexo, ano e região do país.

\section{Método}

Trata-se de um estudo descritivo e exploratório, com dados secundários, obtidos através do Sistema Nacional de Agravos de Notificação (SINAN) e do Sistema de Informação sobre Mortalidade (SIM), 
coletados durante o período de fevereiro e março de 2019. As variáveis investigadas foram: óbitos por intoxicação, faixa etária, agente tóxico, ano, sexo e região do país. O recorte temporal foi feito entre 2007 a 2017 nas faixas etárias ente 15-19 anos, 20-39 anos, 40-59 anos, 60-64 anos e 65-69 anos. A faixa etária entre 70-79 anos não foi incluída porque nesse grupo contém dados a partir dos 76 anos, que é considerada a expectativa de vida do brasileiro em 2017, sendo assim esses óbitos não contam como anos potenciais de vida perdidos.

O cálculo para determinação dos APVP ocorreu em três etapas: I. Foi determinada a idade média dentro de cada faixa etária escolhida; II. Baseando-se na expectativa de vida dos brasileiros, fornecida pelo Instituto Brasileiro de Geografia e Estatística ${ }^{8}$ no ano de 2017 como 76 anos, esta foi considerada a idade predeterminada de morte nesse estudo. Então foi subtraída a "idade média dentro de cada faixa etária" da "idade predeterminada de morte - 76 anos", para obter os anos restantes. III. Por fim, multiplicou-se os anos restantes pelo número de óbitos em cada faixa etária, alcançando assim os APVP por faixa etária.

Devido à utilização de dados secundários para o alcance dos objetivos desse estudo, disponibilizados em bases de dados eletrônicas de domínio público, não houve necessidade de submissão ao Comitê de Ética em Pesquisa, assim como a obtenção de consentimento pelos participantes.

\section{Resultados}

No Brasil, no período de 2007 a 2017, foram registrados pelo SIM 5.662.456 óbitos por intoxicação exógena, nas faixas etárias entre 15 a 69 anos, que resultou em um crescimento da mortalidade proporcional de 2007 a 2011 de 7\%, apresentando uma redução discreta em 2012 e posteriormente retomou o crescimento de mais 7\% dessa mortalidade em 2013. Esse último ano citado foi responsável pelo maior percentual das causas de óbito registrados no país por intoxicação exógena, correspondendo a 19\% (Gráfico 1).

Gráfico 1. Mortalidade proporcional por intoxicação exógena no Brasil no período de 2007 a 2017

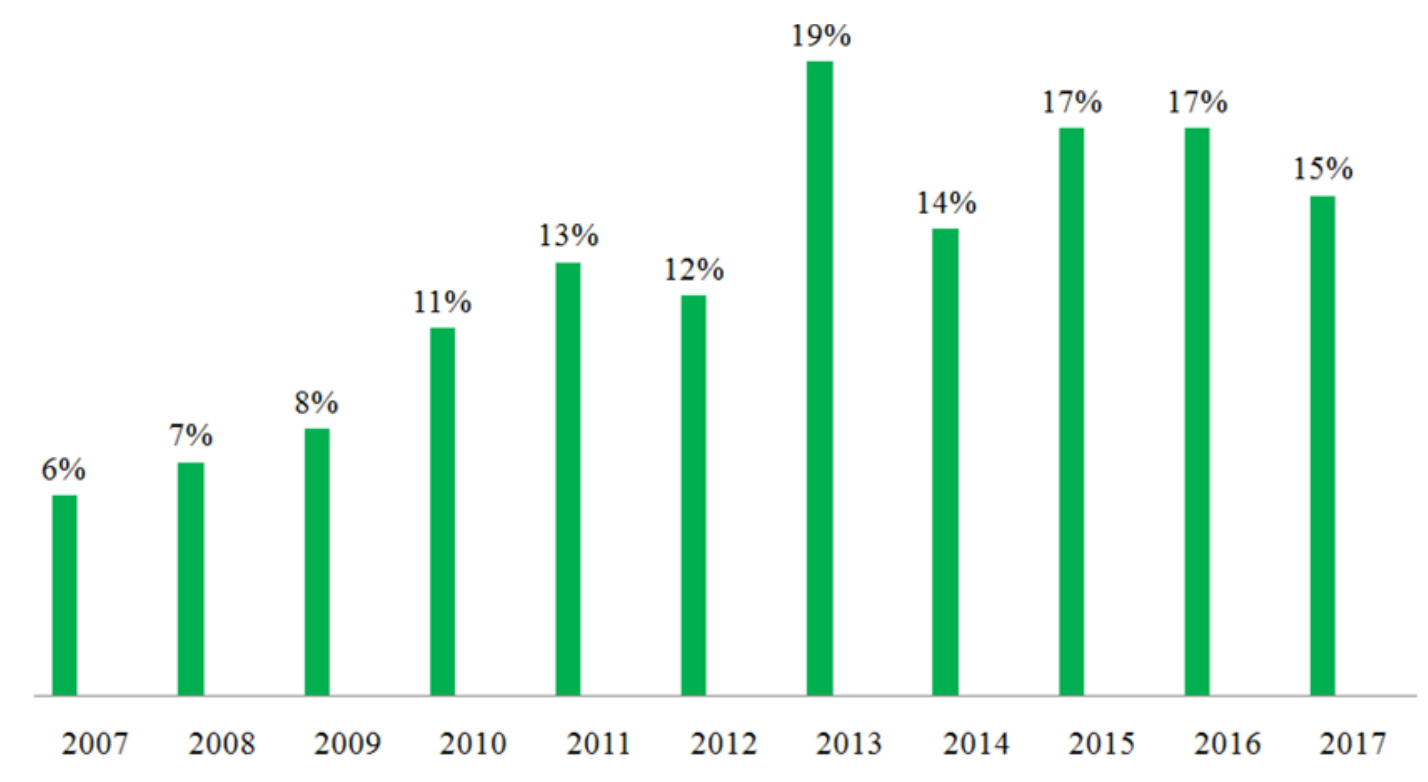

Fontes: $\operatorname{SIM}^{9}$ e $\operatorname{SINAN}^{10}$ (2019). 
O gráfico 2 retrata os Anos Potenciais de Vida Perdidos por faixa etária e sexo no Brasil no período de 2007 a 2017. Com base na análise temporal dos óbitos por intoxicação exógena, observou-se que a faixa etária mais acometida foi a de 20 a 39 anos, sendo responsável por $56,7 \%$ dos casos. Predominou o sexo masculino, que representa $60 \%$ dos casos de morte prematura.

Gráfico 2. Anos Potenciais de Vida Perdidos por faixa etária e sexo no Brasil de 2007 a 2017

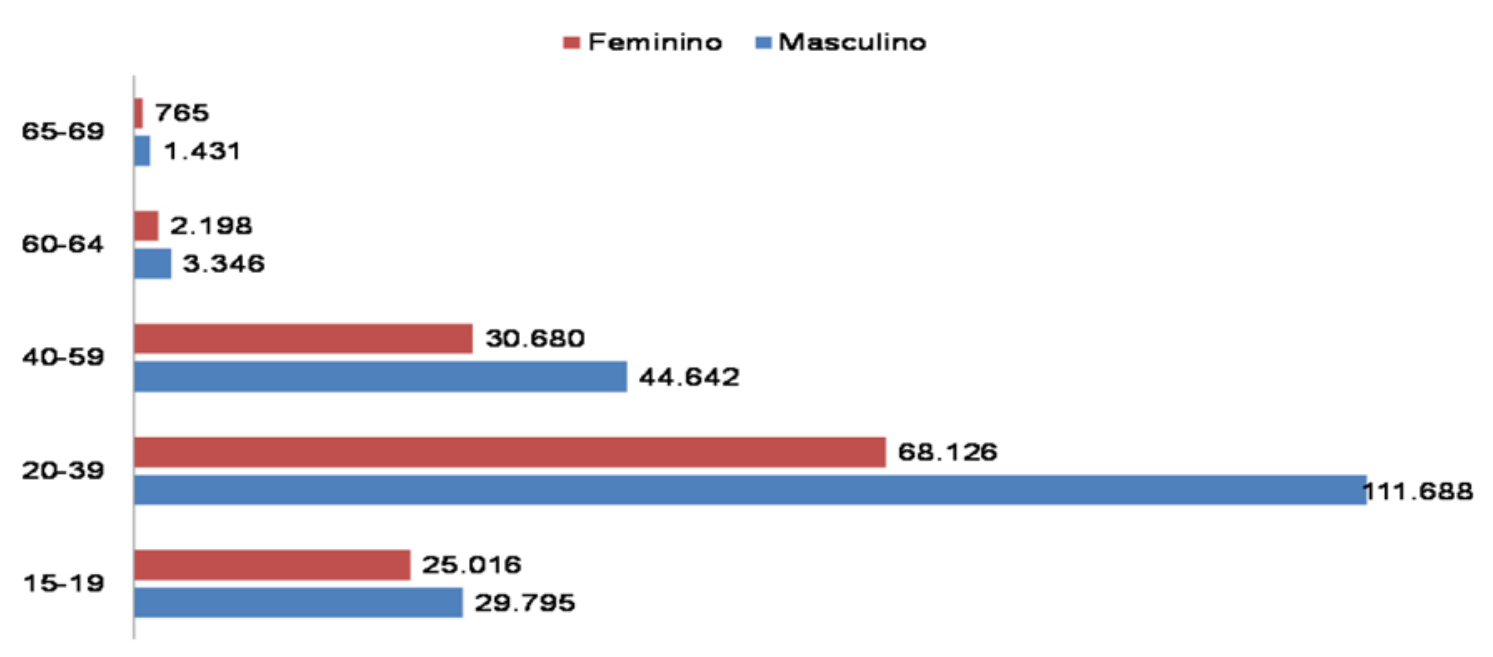

Fonte: SINAN $^{10}$, (2019).

Verificou- se um total de 317.687 APVP entre os anos de 2007 a 2017 no Brasil, onde a faixa etária entre 20 a 39 anos representa $56 \%$ dos óbitos (Tabela 1).

Tabela 1. Anos Potenciais de Vida Perdidos dos 15 aos 69 anos no Brasil no período de 2007 a 2017

\begin{tabular}{ccccc}
\hline Faixa etária & Idade média & Anos restantes & Óbitos & APVP \\
\hline $15-19$ anos & 17 anos & 59 anos & 929 & 54.811 \\
$20-39$ anos & 30 anos & 46 anos & 3909 & 179.814 \\
$40-59$ anos & 50 anos & 26 anos & 2897 & 75.322 \\
$60-64$ anos & 62 anos & 14 anos & 396 & 5.544 \\
$65-69$ anos & 67 anos & 09 anos & 244 & 2.196 \\
\hline
\end{tabular}

Fonte: As autoras (2019).

Em todas as regiões a faixa etária predominantemente mais acometida foi entre 20-39 anos. A região sudeste apresentou maior valor de APVP que outras regiões do país, onde o risco de morte em comparação com o norte é 16 vezes maior, em comparação com o centro-oeste é 8 vezes maior, com o sul 1,9 vezes maior e com o nordeste 1,7 vezes maior (Gráfico 3). 
Gráfico 3. Anos Potenciais de Vida Perdidos distribuídos por faixa etária por região geográfica. Brasil, no período de 2007 a 2017

\begin{tabular}{|c|c|c|c|c|c|}
\hline & & & & & \\
\hline 120.000 & & & & & \\
\hline $\begin{array}{r}100.000 \\
80.000\end{array}$ & & & & & \\
\hline 60.000 & & & & & \\
\hline 20.000 & $\ldots$ & & & L & 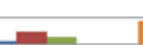 \\
\hline & NORTE & NORDESTE & SUDESTE & SUL & $\begin{array}{l}\text { CENTRO- } \\
\text { OESTE }\end{array}$ \\
\hline 15-19 & 1.534 & 13.511 & 25.724 & 11.918 & 2.124 \\
\hline$=20-39$ & 5.520 & 46.368 & 77.878 & 40.894 & 9154 \\
\hline 40-59 & 1.404 & 17.576 & 33.384 & 18.226 & 4.732 \\
\hline$=60-64$ & 112 & 1.372 & 2.436 & 1.302 & 322 \\
\hline 65-69 & 99 & 612 & 828 & 441 & 216 \\
\hline - TOTAL & 8.669 & 79.439 & 140.250 & 72.781 & 16.548 \\
\hline
\end{tabular}

Fonte: $\operatorname{SINAN}^{10}$, (2019).

Observou-se um crescimento de 280\% nos APVP por agentes tóxicos entre os anos de 2007 a 2013. Associadamente, o uso indevido de medicamentos foi responsável pelo maior número de APVP no país entre os anos de 2007 e 2017, correspondendo a 23,4\%, seguido das drogas de abuso, com $21 \%$, dos agrotóxicos agrícolas, com $19,3 \%$ e dos raticidas, com 12,9\%. Dessa forma, as quatro categorias de agentes tóxicos citadas contribuíram para aproximadamente $76 \%$ dos anos prematuros de vida perdidos e toda sua potencialidade econômica, social e cultural (Tabela 2).

Tabela 2. Anos Potenciais de Vida Perdidos por agente tóxico entre os anos de 2007 a 2017 no Brasil

\begin{tabular}{|c|c|c|c|c|c|c|c|c|c|c|c|c|}
\hline Agente Tóxico & 2007 & 2008 & 2009 & 2010 & 2011 & 2012 & 2013 & 2014 & 2015 & 2016 & 2017 & Total \\
\hline Ign/Branco & 1578 & 1616 & 1828 & 2041 & 2481 & 2183 & 2322 & 1799 & 2681 & 2701 & 3045 & 24275 \\
\hline Medicamento & 1347 & 3617 & 3584 & 5285 & 6707 & 7490 & 7869 & 8977 & 8663 & 9451 & 11141 & 74131 \\
\hline Agrotóxico agrícola & 4798 & 4408 & 5018 & 5760 & 6184 & 4559 & 7435 & 5285 & 5841 & 6409 & 5396 & 61093 \\
\hline Agrotóxico doméstico & 173 & 262 & 282 & 564 & 483 & 548 & 753 & 264 & 360 & 328 & 452 & 4469 \\
\hline Agrotóxico saúde pública & 0 & 0 & 0 & 46 & 0 & 46 & 46 & 118 & 26 & 26 & 0 & 308 \\
\hline Raticida & 2251 & 3087 & 3076 & 4597 & 5101 & 4702 & 3633 & 3656 & 4532 & 3593 & 2803 & 41031 \\
\hline Prod. veterinário & 426 & 171 & 498 & 167 & 675 & 505 & 446 & 421 & 531 & 356 & 501 & 4697 \\
\hline Prod. uso domiciliar & 98 & 223 & 98 & 219 & 533 & 498 & 713 & 567 & 547 & 718 & 709 & 4923 \\
\hline Cosmético & 0 & 0 & 46 & 0 & 0 & 0 & 151 & 72 & 92 & 59 & 14 & 434 \\
\hline Prod. químico & 474 & 259 & 261 & 630 & 780 & 678 & 1186 & 805 & 1294 & 1005 & 900 & 8272 \\
\hline Metal & 0 & 0 & 0 & 92 & 98 & 59 & 151 & 46 & 0 & 0 & 26 & 472 \\
\hline Drogas de abuso & 352 & 1530 & 1530 & 1759 & 2992 & 4527 & 5836 & 8529 & 13202 & 14633 & 11512 & 66402 \\
\hline Planta tóxica & 0 & 0 & 46 & 40 & 35 & 138 & 0 & 46 & 0 & 46 & 166 & 517 \\
\hline Alimento e bebida & 138 & 92 & 302 & 274 & 924 & 309 & 759 & 676 & 671 & 649 & 855 & 5649 \\
\hline Outro & 236 & 524 & 428 & 376 & 540 & 565 & 13832 & 839 & 856 & 927 & 1154 & 20277 \\
\hline Total & 11871 & 15789 & 16997 & 21850 & 27533 & 26807 & 45132 & 32100 & 39296 & 40901 & 38674 & 316950 \\
\hline
\end{tabular}

Fonte: SINAN $^{10}, 2019$.

Ao longo do período investigado, verificou-se um aumento importante dos óbitos por drogas de abusos que chegaram a ultrapassar o número de óbitos por medicamentos nos anos de 2015, 2016 e 2017 e também o número de óbitos por agrotóxico agrícola nos anos de 2014, 2015, 2016 e 2017. 


\section{Discussão}

A estimativa dos anos potenciais de vidas perdidos a partir de dados secundários sobre óbitos permitiu observar uma tendência crescente quanto à mortalidade proporcional pelas intoxicações exógenas no Brasil de 2007 a 2017, com maiores percentuais a partir de 2013.

No Brasil, no período de 2007 a 2017, os óbitos por intoxicação exógena se sobressaíram na população masculina. Em geral as mulheres vivem, em média, de seis a oito anos a mais que homens. Esta diferença deve-se principalmente a uma vantagem biológica inerente as mulheres, mas também as diferenças comportamentais entre homens e mulheres ${ }^{11}$.

O suicídio não é tão comum entre as mulheres devido a baixa prevalência de alcoolismo, a religiosidade, as atitudes flexíveis em relação às aptidões sociais e ao desempenho de papéis durante a vida. Além disso, as mulheres reconhecem mais cedo sinais de risco para depressão, suicídio e doença mental, buscam ajuda em momentos de crise e participam nas redes de apoio social. Também, os métodos utilizados na tentativa de suicídio diferem entre os sexos. Os homens tendem a usar meios mais violentos do que as mulheres devido a uma maior intenção suicida, a agressividade, ao conhecimento sobre esses meios e a uma menor preocupação com a desfiguração do corpo, enquanto as mulheres tendem a tomar doses excessivas de substâncias ou de venenos, no entanto isso não pode ser considerado uma regra ${ }^{12}$. Além do envolvimento com atividades ocupacionais que tornam mais fácil o acesso a produtos tóxicos à saúde ${ }^{13}$.

Com relação ao sexo e a faixa etária tem-se que a mortalidade masculina apresenta maior magnitude em todas as faixas etárias, com destaque entre 20 a 59 anos, sendo mais expressivo entre 20 e 39 anos de idade. Essa concentração de óbitos prematuros expressa impacto econômico e social no país, pela perda da vida produtiva, além do impacto emocional e psicológico para as famílias que perdem seus entes queridos ${ }^{11}$.

O estudo evidenciou um número importante de anos potenciais de vida perdidos por uso inadequado de medicamentos ${ }^{14}$. O Ministério da Saúde em 2018 fez um levantamento que aponta que a intoxicação exógena é o meio utilizado por mais da metade das tentativas de suicídio notificadas no país. Com relação aos óbitos, a intoxicação é a segunda causa, com $18 \%$, ficando atrás das mortes por enforcamento, que atingem 60\% do total. Entre 2007 e 2016, foram registrados no Sistema de Informações sobre Mortalidade (SIM) 106.374 óbitos por suicídio. Em 2016, a taxa chegou a 5,8 por 100 mil habitantes, com a notificação de 11.433 mortes por essa causa. As mulheres representaram quase 70\% (153.745) do total de tentativas de suicídio por intoxicações exógenas no período de 2007 a 2018. Sobre os agentes tóxicos utilizados, os medicamentos correspondem a $74,6 \%$ das tentativas entre as mulheres e $52,2 \%$ entre os homens. As intoxicações exógenas resultam em 4,7\% de óbitos em homens e 1,7\% nas mulheres ${ }^{15}$.

O raticida destacou-se entre os agentes tóxicos mais notificados, estando relacionado a acidentes domésticos graves e a tentativas de suicídio. Cerca de $90,4 \%$ dos casos são tentativas de suicídios ou suicídios e 9,6\% acidente individual ${ }^{16}$. Segundo a Agência Nacional de Vigilância Sanitária (ANVISA), o uso irregular e indiscriminado do raticida no país e o fácil acesso nos centros urbanos, acarreta um grave problema de saúde pública ${ }^{17}$.

As drogas de abuso, relacionadas ou não a tentativa de suicídio, também têm sido responsáveis por um número importante de anos potenciais de vida perdidos. São drogas que afetam a atuação do sistema nervoso central, podendo causar alterações de humor, e/ou diminuição do nível de percepção ${ }^{18}$. O uso de drogas constitui um sério problema de saúde pública. As emoções e sofrimento psíquico acentuado, como por exemplo em casos de depressão, déficit na autoestima, elevado grau de ansiedade, são fatores que predispõe o uso das drogas de abuso ${ }^{19}$.

Acredita-se que o aumento de óbitos por suicídio, e relacionado a isso, o aumento de casos de intoxicações exógenas visando este fim, esteja relacionado a depressão. A Organização Mundial de Saúde apontou em seu relatório global correspondente ao período de 2005 a 2015, um aumento no número de casos de depressão em 18\%, sendo em sua maioria do sexo feminino. 0 mesmo estudo indicou que $5,8 \%$ dos brasileiros sofrem por depressão ${ }^{20}$. 
As diferenças geográficas presentes no Brasil convergem também na análise dos Anos Potenciais de Vida Perdidos. O Sudeste, Nordeste, Sul, Centro-Oeste e Norte representam nessa ordem do maior ao menor valor dos APVP. As regiões que representam o primeiro, o terceiro e o quarto maior valor de mortes prematuras são regiões de alto Índice de Desenvolvimento Humano (IDH) e Índice de Desenvolvimento Humano Municipal (IDHM) em longevidade e renda de alto a acima da média para os valores nacionais. Já o Nordeste e Norte, segundo e quarto colocados em APVP por intoxicação exógena, respectivamente, apresentam um Alto IDHM de $90,2 \%$ e $71 \%$, respectivamente, e o IDHM Renda classificado como médio ${ }^{21}$.

\section{Conclusão}

Houve uma perda de 317.687 APVP, entre 2007 a 2017, onde a faixa etária entre 20 a 39 anos representou $56 \%$ dos óbitos. Desse recorte temporal o ano de 2013 destacou- se com a mortalidade proporcional por intoxicação exógena que alcançou 19\% das mortes no país, mesmo sendo esta uma causa prevenível. Destas mortes prematuras o sexo masculino representa $60 \%$ dos APVP quando comparado com o sexo feminino. A região sudeste apresentou maior valor de APVP que outras regiões do país, onde o risco de morte em comparação com o norte é 16 vezes maior.

Os agentes tóxicos mais prevalentes nas intoxicações exógenas registradas foram os medicamentos, drogas de abusos, agrotóxico agrícola e raticida respectivamente, totalizando mais da metade de APVP do período. A utilização inadequada dos medicamentos foi responsável pelo maior número de óbitos, sendo o agente tóxico mais utilizado nas tentativas de suicídio.

O aumento dos casos de morte prematura por intoxicação exógena reforça a importância do trabalho da vigilância em saúde, pois para o conhecimento do nível de saúde da população, é preciso ter o entendimento das tendências a fim de que haja devido planejamento e implementação de programas de saúde e medidas para prevenção dos agravos. Os indicadores são os principais aliados desse processo, por isso, vale ressaltar que a qualidade das informações depende da precisão e alimentação dos sistemas de informação.

Multifacetada são as medidas que podem ser adotadas para tratar essa questão prevenível, dos APVP por intoxicação exógena, iniciando pela identificação das situações de exposição a situações de risco, perpassando por vigilância participativa, busca ativa, educação em saúde, articulações intersetoriais, notificações e medidas de prevenção das reincidências.

Ressalta-se como limitação desse estudo a coleta de dados em plataforma de domínio público, alimentada pelos serviços de saúde, podendo ter ocorrido subnotificação dos casos de intoxicação exógena no país.

\section{Contribuições dos autores}

Faustino TN participou da concepção, delineamento, análise estatística dos dados da pesquisa, interpretação dos resultados e redação do artigo científico. Maia SS, Souza VS e Souza ED participaram da concepção, delineamento, busca e análise estatística dos dados da pesquisa, interpretação dos resultados e redação do artigo científico.

\section{Conflitos de interesses}

Nenhum conflito financeiro, legal ou político envolvendo terceiros (governo, empresas e fundações privadas, etc.) foi declarado para nenhum aspecto do trabalho submetido (incluindo mas não limitando-se a subvenções e financiamentos, participação em conselho consultivo, desenho de estudo, preparação de manuscrito, análise estatística, etc.). 


\section{Referências}

1. Ministério da Saúde. Secretaria de Vigilância em Saúde. Coordenação- geral de desenvolvimento da epidemiologia em serviços. Guia de vigilância em saúde: volume único. [Internet]. 2017. [Acesso em 11 mar. 2019]. Disponível em: http:// portalarquivos.saude.gov.br/images/pdf/2017/outubro/06/ Volume-Unico-2017.pdf

2. Organização Mundial de Saúde. Poisoning Prevention and Management. [Internet]. 2019. [Acesso em 11 mar. 2019]. Disponível em: https://www.who.int/ipcs/poisons/en/

3. Ministério da Saúde. Perfil epidemiológico das tentativas e óbitos por suicídio no Brasil e a rede de atenção à saúde. [Internet]. Ministério da Saúde; 2017. [Acesso em 10 set. 2018]. Disponível em: http://portalarquivos2.saude.gov.br/images/ pdf/2017/setembro/21/2017-025-Perfil-epidemiologico-dastentativas-e-obitos-por-suicidio-no-Brasil-e-a-rede-de-atencao-asaude.pdf

4. Departamento de Informática do SUS. DATASUS. [Internet]. 2019. [Acesso em 05 mar. 2019]. Disponível em: http://tabnet. datasus.gov.br/cgi/tabcgi.exe?sinannet/cnv/Intoxbr.def

5. Peixoto HCG, Souza ML. O indicador Anos Potenciais de Vida Perdidos e a ordenação das causas de morte em Santa Catarina. Informe Epidemiológico do SUS 1999; 8(1): 17-25. doi: $10.5123 /$ S0104-16731999000100003

6. Bochner R, Freire MM. Análise dos óbitos decorrentes de intoxicação ocorridos no Brasil de 2010 a 2015 com base no Sistema de Informação sobre Mortalidade (SIM). Cien Saude Colet. 2018 ago. [Acesso em 10 fev. 2019]. Disponível em: http:// www.cienciaesaudecoletiva.com.br/artigos/analise-dos-obitosdecorrentes-de-intoxicacao-ocorridos-no-brasil-de-2010-a2015-com-base-no-sistema-de-informacao-sobre-mortalidade$\operatorname{sim} / 16908$ ?id $=16908$

7. Gordis L. Ocorrência de doenças: II. Mortalidade e outras medidas de impacto de doenças. In: Gordis L. Epidemiologia. 5. ed. Thieme Revinter Publicações: Rio de Janeiro; 2017. p.61-87.

8. Instituto Brasileiro de Geografia e Estatística. Em 2017, expectativa de vida era de 76 anos. [Internet]. 2019. [Acesso em 10 fev. 2019]. Disponível em: https://agenciadenoticias.ibge. gov.br/agencia-sala-de-imprensa/2013-agencia-de-noticias/ releases/23200-em-2017-expectativa-de-vida-era-de-76-anos

9. Ministério da Saúde. Sistema de Informação de Mortalidade (SIM). [Internet]. 2019. [Acesso em 10 fev. 2019]. Disponível em: http://tabnet.datasus.gov.br/cgi/tabcgi.exe?sim/cnv/obt10uf.def

10. Ministério da Saúde. Sistema de Informação de Agravos de Notificação (SINAN). [Internet]. 2019. [Acesso em 11 mar. 2019]. Disponível em: http://tabnet.datasus.gov.br/cgi/tabcgi. exe?sinannet/cnv/Intoxbr.def
11. Organização Mundial de Saúde. Mulheres e saúde: evidências de hoje, agenda de amanhã. [Internet]. 2009. [Acesso em 11 mar. 2019]. Disponível em: https:// who.int www. ageing/mulheres_ saude.pdf /

12. Vieira LP, Santana VTP, Suchara EA. Caracterização de tentativas de suicídios por substâncias exógenas. Cad. Saúde Colet. 2015;23(2):118-123. doi: 10.1590/1414-462X201500010074

13. Reis LM, Martins BF, Gavioli A, Mathias TAF, Oliveira MLF. Saúde do homem: internações hospitalares por intoxicação registradas em um centro de assistência toxicoló. Esc Anna Nery. 2013;17(3):5005-511.

14. Matos KF, Martins CBG. Perfil epidemiológico da mortalidade por causas externas em crianças, adolescentes e jovens na capital do Estado de Mato Grosso, Brasil, 2009. Epidemiol. Serv. Saúde.2012;21(1):43-53. doi: 10.5123/S1679-49742012000100005

15. Fundação Osvaldo Cruz. Novos dados do MS reforçam importância da prevenção do suicídio. [Internet]. 2019. [Acesso em 14 jun. 2019]. Disponível em: https://agencia.fiocruz.br/novosdados-do-ms-reforcam-importancia-da-prevencao-do-suicidio

16. Martin BF, Hungaro AA, Santos JAT, Meschia WC, Correia LM, Oliveira MLF. Intoxicação por raticida em um Centro de Assistência Toxicológica. Revista da Rede de Enfermagem do Nordeste. 2016;17(1):3-9. doi: 10.15253/21756783.2016000100002

17. Ministério da saúde. Agencia nacional de vigilância sanitária (ANVISA). Nota técnica da reavaliação do ingrediente ativo aldicarbe. [Internet]. 2019. [Acesso em 12 jun. 2019] Disponível em: http://portal.anvisa.gov.br/ documents/111215/117797/aldicarbe.pdf/1e2e3d0b-cfe5-4d5cad4d-f3bc58dca316?version=1.0

18. Raupp L, Adorno RCF. Circuitos de uso de crack na região central da cidade de São Paulo (SP, Brasil). Cienc Saude Colet 2011;16(5):2613-22. doi: S143-81232011000500031

19. Marques ACPR, Cruz MS. O adolescente e o uso de drogas. Rev. Bras. Psiquiatr. 2000;22(2):32-6. doi: S151644462000000600009

20. Organização Mundial de Saúde. Depressão e outros distúrbios mentais comuns: estimativas globais de saúde. [Internet]. 2017. [Acesso em 14 Jun. 2019]. Disponível em: https://nacoesunidas. org/oms-registra-aumento-de-casos-de-depressao-em-todo-omundo-no-brasil-sao-115-milhoes-de-pessoas/

21. Ministério do Trabalho e Emprego. Instituto de Pesquisa Econômica Aplicada (IPEA). Desenvolvimento humano nas macrorregiões brasileiras. [Internet]. IPEA: Brasília; 2016. [Acesso em 25 maio 2019]. Disponível em: http://www.ipea.gov.br/portal/ images/stories/PDFs/livros/livros/20160331_livro-idhm.pdf 